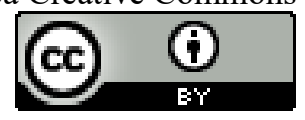

\title{
TERRITÓRIO EXISTENCIAL AFROBOLIVIANO OU UMA CONCEPÇÃO TOCAÑERA DA PACHAMAMA
}

Resumo: Partindo da maneira tocañera (habitante da comunidade afroboliviana de Tocaña) de ordenar o mundo em comum, o trabalho a seguir pretende percorrer os territórios (meta)físicos habitados por aqueles que pertencem à comunidade afroboliviana de Tocaña. As ideias apresentadas aqui são o produto do meu processo de convivência e aprendizado que empreendi com os tocañeros nos anos 2015 e 2016. A partir de um evento etnográfico que ocorreu em 2015, as idéias incorporadas aqui giram em torno do complexo conceito da Pacha, o qual é (re)criado a todo momento pelos tocañeros. Com o objetivo de aproximar o leitor da noção da Pacha (usualmente traducido como "tierra" o "mundo"), procurei estabelecer diálogos entre conceitos extraídos de duas propostas ontológicas que, do meu ponto de vista, compartilham características metafísicas em segundo plano: a ontologia tocañera e a ontologia Whiteheadiana.

Palavras-Chave: Afrodescendentes; Negritude; Bolivia; Pachamama; Giro ontológico.

\section{AFROBOLIVIAN EXISTENTIAL TERRIORIES OR THE TOCAÑEROS' CONCEPTION OF PACHAMAMA}

\begin{abstract}
Departing from the way the tocañeros order the world in common, this work pretends to elaborate on the (meta)physical territories dwelled by those who belong to the Afrobolivian Community of Tocaña. The ideas presented here are the product of my coexistence, and learning process undertook, with the Tocañeros during the years 2015 and 2016. Focusing on an ethnographic event that occurred in July 2015, the ideas embodied here revolve around the complex concept of Pacha that the tocañeros keep on (re)elaborating at all times. With the aim of bringing the reader closer to the tocañeros' perception of Pacha, I have tried to generate dialogues between concepts taken from two ontological proposals that, in my view, share in their depths some metaphysical precepts: the tocañeros' ontology and whiteheadian ontology.
\end{abstract}

Key-words: Afrodescendants; Blackness; Andes; Pachamama; Bolivia; Ontological turn.

\footnotetext{
${ }^{1}$ Graduação "cum laude" em Antropologia e Ciência Política pelo College of Idaho (EUA) em 2013, Magister "Cum laude" em Antropologia Social pela Universidade Federal do Rio Grande do Sul (Brasil) na 2016, Magister "cum laude" em Estudos Latino-Americanos pela Universidade de Amsterdã (Holanda) em 2017 e atualmente é doutorando no programa de Antropologia Social do Museu Nacional do Brasil Universidade Federal do Rio de Janeiro. E-mail: luisreyes.2703@gmail.com
} 


\section{Otensonsen}

$\triangle \nabla \Delta \nabla \Delta \nabla \Delta \nabla \triangle \nabla \nabla \nabla \nabla \Delta \nabla \Delta \nabla \Delta \nabla \Delta \nabla \Delta \nabla \Delta \nabla \nabla \Delta \nabla \Delta \nabla \Delta \nabla \Delta \nabla \Delta \nabla \nabla \nabla \nabla$

TERRITORIO EXISTENCIAL AFROBOLIVIANO O UMA CONCEPCIÓN

TOCAÑERA DE LA PACHAMAMA

Resumen: Partiendo de la forma tocañera de ordenar el mundo en común, el siguiente trabajo pretende discurrir sobre los territorios (meta)físicos habitados por aquellas personas que pertenecen a la comunidad afroboliviana de Tocaña. Las ideas aquí expuestas son producto de mi convivencia y proceso de aprendizaje que emprendí con los tocañeros en los años 2015 y 2016. A partir de un evento etnográfico ocurrido en el 2015, las ideas aquí plasmadas giran en torno a la complejo concepto de la Pacha que los tocañeros (re)creado a cada momento. Teniendo como objetivo acercar al lector a la noción tocañera de la Pacha, es que he intentado generar diálogos entre conceptos tomados de dos propuestas ontológicas que, desde mi punto de vista, comparten características metafísicos fundamentais: la ontología tocañera y la ontología whiteheadiana.

Palabras-clave: Afrodescendientes; Negritud; Andes; Pachamama; Bolivia; Giro ontológico.

\section{LE TERRITOIRE EXISTENTIEL AFROBOLIVIEN OU UNE CONCEPTION TOCAÑERA DE PACHAMAMA}

Résumé: Partant de la façon tocañera d'organiser le monde en commun, le suivant travail cherche parcourir les territoires (méta)physiques habités par ceux qui appartiennent à la communauté afro-bolivienne de Tocaña. Les idées présentées ici sont le produit de mon processus de coexistence et d'apprentissage que j'ai entrepris avec les tocañeros dans les années 2015 et 2016. À partir d'un événement ethnographique qui a eu lieu en 2015, les idées incorporées ici tournent autour du complexe concept de Pacha, lequel il est (re)créé à tout moment par les tocañeros. Afin de rapprocher le lecteur de la notion de Pacha (généralement traduit par «terre» ou «monde»), j'ai essayé d'établir des dialogues entre des concepts extraits de deux propositions ontologiques qui, du mon point de vue, partagent des caractéristiques métaphysiques en arrière-plan: l'ontologie tocañera et l'ontologie de Whitehead.

Mots-clés: Afro-descendants; Négritude; Bolivie; Pachamama; Virage ontologique.

\section{INTRODUCCIÓN}

El siguiente texto adopta la forma de una narrativa etnográfica a partir de la cual se propone internarnos en los diversos cohabitares establecidos entre la Pachamama y los pobladores de la comunidad afroboliviana de Tocaña, con quienes tuve la oportunidad de convivir durante partes de los años 2015 y 2016. Tocaña es una comunidad compuesta por un aproximado de treinta familias, en su mayoría afrobolivianas y algunas pocas familias aymaras, que se ubica en una montaña al frente de Coroico (capital de la 
provincia de Nor-Yungas del departamento de La Paz) ${ }^{2}$. A partir de un evento etnográfico ocurrido en el 2015, las ideas plasmadas en los siguientes párrafos buscan discurrir sobre el carácter múltiple que la Pachamama tiene para los tocañeros. Es así que, entrelezando eventos etnográficos e ideas propuestas por mi cohabitar con los tocañeros con conceptos provenientes de una lectura especulativa de la ontología realista whiteheadiana, se encarará a la Pacha no sólo como ser extrahumano con quien se cohabita sempiternamente ni tampoco como concepto metafísico el cual nos remite a los territorios existenciales de los afrobolivianos. Partiendo y caminando de la mano con el pensamiento tocañero, en este texto pretendo explorar el polo especulativo de la Pachachama el cual la coloca como territorio existencial (concepto guattariano que evoca la manifestación incorpórea de universos a través de los que el yo se conecta con el ambiente) y como un plano de imanencia, es decir, un manto vacío en el que, por el hecho de estar vacío, aun todo existe como posible.

\section{EL PEROLANI: PUERTA CÓSMICA A LA PACHA}

Agosto del 2015. El Perolani, la montaña más grande, alta, con vegetación más densa, guarda en sus entrañas árboles de toda importancia que los pobladores de la comunidad afroboliviana de Tocaña usan para elaborar los tambores de la saya y herramientas domésticas. En él también se refugian diversas plantas medicinales y animales salvajes y, en su corazón que llena de vida a la montaña y a todos los seres que la habitan, se encuentra una poderosa cascada, fuente de agua más preciada de los alrededores y vertiente mítica del río Coroico.

Durante mi estancia en Tocaña aprendí que los tocañeros acostumbran adentrarse en el Pelorani sólo cuando es imprescindible hacerlo ya que las fuerzas del bosque pueden tragarlos, así como un agujero negro traga estrellas en el espacio, sin devolverlos de la forma como eran antes. Sólo los niños y adolescentes osan adentrarse sin mucho cuidado, desafiando los consejos de los adultos de la comunidad. Este cuidado debe de ejercerse ya que, más que un simple bosque, el Perolani es la otra cara del mundo de aquí; es un

\footnotetext{
${ }^{2}$ Link de la ubicación de Tocaña y Coroico:

$<$ https://www.google.com/maps/place/Paraiso+de+Toca\%C3\%B1a+Camping/@,-16.1590891,$67.7410378,14.52 \mathrm{z} / \mathrm{data}=! 4 \mathrm{~m} 5 ! 3 \mathrm{~m} 4$ ! $1 \mathrm{~s} 0 \mathrm{x} 915 \mathrm{f} 7365 \mathrm{bb} 1 \mathrm{f} 75 \mathrm{c} 7: 0 \mathrm{xc} 26 \mathrm{~b} 5 \mathrm{e} 2 \mathrm{c} 4008647 \mathrm{a} ! 8 \mathrm{~m} 2 ! 3 \mathrm{~d}-$ $16.1674275 ! 4 \mathrm{~d}-67.7296561>$
} 
mundo en donde también las almas, espíritus y dioses habitan. Por ello, hay que tener cuidado si uno va al Perolani, ya que en ese bosque no solo nos ponemos a merced de animales peligrosos sino también seres del mundo de abajo (también llamado "otro mundo") y, menos a menudo, del mundo de arriba (también denominado "cielo").

Según me contaron algunos tocañeros, en lugares tan inhóspitos como el denso bosque del Perolani es donde les gusta vivir a seres fácilmente irritables como El Tío o El Mallku, a seres malignos como los anchanchu (demonios) o la tiyula (la llorona); incluso, a veces, se encuentran seres todopoderosos como la Pachamama o el Kunturi Mamani, corporificados en diferentes formas.

Un cálido día de Julio del 2015, bajo un sol abrazador, un pequeño grupo de personas conformado por tres tocañeros (tío Mastuco, Dennis y Diego), dos argentinos (Luciano y Sebastián) y yo (peruano), nos encaminamos con dirección al Perolani. Salimos junto con el sol para regresar, con total seguridad, antes de su puesta. Cada uno de nosotros cargaba un machete, mientras tío Mastuco, el más viejo del grupo, adicionalmente preparó un morral en el que guardó una botella de alcohol Ceibo de 95 grados, cigarrillos, y una bolsa plástica llena de hojas de coca. Ilusamente, pensé que el alcohol y lo demás era para nosotros y, sabiendo que los tocañeros, cuando no hay otra opción, beben una mezcla de alcohol de 95 con agua, escondí en mi morral una botellita de guarapo que tía Raymunda me había obsequiado.

Mientras subíamos por la montaña, le dije a Diego, casi susurrando, que tenía guarapo para mezclar con el alcohol. Con cara de extrañamiento me miró y me dijo, "pero si el trago no es para nosotros. Es para El Tío. Allá no se puede hacer locuras. Esa es tierra del Tío. Es bien celoso, y seguro que nos va a estar mirando”. Avergonzado por mi ignorancia, seguí caminando junto a ellos sin hablar demasiado. Subimos sin parar por casi una hora, pasando por cafetales, naranjales y cocales. De vez en cuando veíamos a alguien que nos observaba y preguntaba dónde íbamos. "Al Perolani, a traer palos", respondía Mastuco, mientras desfilábamos en fila india por los estrechos senderos que nos condujeron hasta la puerta del bosque, la cual es el espacio que divide la cara pelada de la montaña habitada por los humanos de la cara copiosa de la montaña la cual es habitada por animales y espíritus de toda clase.

Tío Mastuco nos advirtió que ya era hora de estar más atentos, que los juegos se habían acabado. Esto se debía al hecho de que en la cara donde habitan los humanos, el 
punto de vista de ellos es el más fuerte. Sin embargo, una vez que se cruza al bosque, si no se tiene el cuidado debido, nos podemos perder o volver locos, ya que se nos puede meter un espíritu o un animal nos puede llevar a seguir sus pasos; en otras palabras, podemos adoptar el punto de vista de los otros, adquiriendo, consecuentemente, una forma de ordenar el mundo que los humanos percibimos como anormal.

A los pocos, ingresamos en las entrañas del bosque y sentí que caminamos tanto que nuestras camisetas se empaparon de nuestros sudores. Recuerdo también que mientras más nos adentrábamos, menos podía apreciar el sol y mi húmeda piel sentía que el aire que nos tocaba se comenzaba a volver un tanto más frío. Y así, después de casi una hora de caminata, llegamos a un lugar que Mastuco señaló como el indicado. La densa vegetación permitía el ingreso de sólo unos cuantos rayos de sol. "Por eso está más frío que afuera", pensé. Mastuco, Diego y Denis, con las manos en la cintura, miraban las copas de los árboles,mientras que yo aprovechaba el momento para recuperar el aliento. En la brevedad de ese instante, noté que el lugar donde nos habíamos sentado era muy verde, frío, y silencioso. Además del trino de los pájaros, sólo se lograba escuchar, a lo lejos, el bravo cantar del río Coroico, el cual subía rápidamente de las faldas hasta la cima de la montaña.

“Aquí es más frío porque ya es casi otro mundo", me decía Diego en voz baja mientras tío Mastuco recalcaba que nuestra tarea era buscar troncos rectos y delgados que sirvan para fabricar escaleras. La afirmación de Diego me dejó pensando. Nos sentamos sin hablar por unos minutos, tan sólo mirábamos los árboles, delgados, altísimos, con una copa nada copiosa, unos más rectos que otros, de corteza blanquecina. Tío Mastuco se levantó, abrió la botella de alcohol, y comenzó a esparcirlo en la zona donde nos encontrábamos. Al finalizar, bebió un trago, y nos dijo "esto se hace para que el Tío nos permita ver los árboles con mejor tronco". Después, nos ofreció a todos un poquito de alcohol para beber, y nos dijo que hiciéramos lo mismo. Todos brindamos con el Tío. También nos pasó la bolsa de plástico de la cual teníamos que coger un puñado de hojas de coca. Nos advirtió, a los argentinos y a mi, que dejemos unas cuantas para el Tío. Sacó un trapo, lo acomodó cuidadosamente en la tierra y sobre él puso un par de cigarros. Nos indicó que pusiéramos algunas hojas de coca mientras abría un pequeño hueco en la tierra. Allí enterró la pequeña mesa (ofrenda) diciendo que podíamos iniciar la búsqueda de árboles. 
Me explicaron que esa ofrenda era para el Tío. Cuando les pregunté qué o quién era el Tío, me dijeron que era un tipo de patrón, dueño de ese lugar. Era un especie de santo al cual se le rinde un tributo. La diferencia con los santos católicos, resaltó Mastuco, es que no nos veía desde el cielo, sino que él estaba allí, dando vueltas como un patrón de hacienda. El Tío, a diferencia de los santos que se nos aparecen en visiones tal y como son, nunca revela su aspecto físico a las personas comunes. Tan sólo se muestra tal y como es a los animales, las plantas, y a algunos chamanes. "Por eso es bueno venir con perros, ellos sí lo ven y ladran. Nos avisan”, me explicaría posteriormente Diego.

Sin embargo, a pesar de que nosotros, humanos, no lo veamos, el Tío sí nos ve y, además, conversa con todo aquello que habita en sus tierras y, así, se entera de todo lo que ocurre en sus dominios. Es por ello que si tomas alguna planta o animal sin pedirle permiso, corres el riesgo de que el Tío se interponga en tu camino y evite que le robes, o si le llegas a robar, puede ser que en algún momento tú o tu familia sean castigados. Pero en el Perolani, el Tío no es el único al que hay que tener en cuenta. "El Tío le avisa a la Pacha, y ella ya sabrá cuando te regresa lo que has hecho de vuelta", dijo Mastuco mientras metía más hojas de coca en su boca. "En realidad aunque El Tío es poderoso, a la que hay que tenerle bastante respeto es a la Pachamama", complementó Dennis.

"La Pachamama es como la virgen, pero una virgen que también es como dios... y me había olvidado, pero es más difícil de explicar, porque la Pacha también es la tierra", me explicaba tía Raymunda. La Pachamama, como virgen, es persona, concebida y representada de múltiples maneras. La Pachamama, como dios, todo lo sabe y todo lo puede, es el inicio y el fin, es el eterno tiempo de las transformaciones. La Pachamama, como tierra, es espacio, es universo, es física y metafísica. La Pachamama, al fin de cuentas, comprendí, es una y múltiple. Y el Perolani, me explicaron, es uno de esos lugares donde puedes sentirla en sus diferentes formas al mismo tiempo. Esto es posible ya que el Perolani es un pliegue cósmico, umbral de mundos múltiples, espacio donde uno experimenta a la Pachamama como sagrada o, mejor dicho, es uno de esos espacios en donde la Pachamama, como evento, nos torna sujetos de experiencias.

Los tocañeros me explicaron que no podían definir a la Pachamama de forma concisa ya que sus awichos (abuelos) no la conocían como tal cuando habitaban África. Los ancestros de los tocañeros sólo la conocieron como Pachamama a través de los aymarás y quechuas que ya habitaban los andes y yungas Bolivianos. Sin embargo, según 
la tía Martha Barra, la Pachamama ya era conocida en todo el mundo, desde siempre, porque ella era el mundo y aun más. La única diferencia es que en otros lugares tiene otros nombres y otras maneras de concebirla. Por eso, los negros de los yungas bolivianos la conciben de forma diferente a los aymarás o quechuas, lo que no significa que estén hablando de una Pachamama diferente ni que no entiendan lo que ella es, tan solo significa que hablan de la misma Pachamama, observada desde un punto de vista localizado en un lugar diferente de ella misma.

\section{LA BIFURCACIÓN DE LA PACHA}

Cuando a los huaironcos (extranjeros) se les explica lo que es la Pacha, lo hacen mediante el uso de una dualidad: (1) La Pacha que vemos y pensamos, y (2) La Pacha que tocamos y habitamos. El primer polo de esta dualidad lo percibimos con nuestros ojos y pensamiento de gente. A lo lejos, por ejemplo, con nuestros ojos de gente vemos las verdes y tersas montañas de los Yungas y pensamos en lo bonito y suave que es ese extenso manto de verde terciopelo que las cubre. El segundo polo lo percibimos con nuestro cuerpo, el cual nos conecta a la Pacha. De cerca, en nuestro cuerpo de carne, ese mismo manto de verde terciopelo nos quita y nos llena de energía vital, nos pide trabajo y nos da comida, nos deja cayos en las manos, dolor y placer en el cuerpo. En suma, el primer polo nos remite al alma ... a lo intangible... mientras que el segundo, al cuerpo... a lo material. Es que quinientos años de contacto los han hecho conscientes de que, para que los blancos entiendan, se les debe de explicar las cosas en oposiciones duales como cuerpo/alma. Sin embargo, aunque en la epidermis del argumento se reifique la dualidad, en sus profundidades se mantiene la complejidad propia del pliegue cósmico.

Un primer paso para entrar en las profundidades del pliegue, me enseñaron los tocañeros, es aprender que, de forma aparente, hay dos Pachas. Una Pacha es la que está fuera de nosotros, es aquello que conocemos como medio, ambiente, o mundo exterior; esa pacha es toda aquella materialidad causante de nuestra percepción. Esta primera Pacha puede ser definida, usando la jerga whitehediana, como una naturaleza causal. Es decir, aquella naturaleza primaria que conduce a nuestra mente hacia la percepción. Esta Pacha es, por lo tanto, ese entramado de lo infinitesimal (átomos, moléculas y sustancias) que nos permite percibir, por ejemplo, el aterciopelado manto verde que se extiende sobre las 
montañas yunqueñas. La otra Pacha, en cambio, se manifiesta en cada una de nuestras percepciones; colores, sabores, olores, temperatura, todo aquello que nos hace saber que en ella, de facto, seguimos habitando. Continuando con el diálogo con Alfred $\mathrm{N}$. Whitehead, podría decirse que esta segunda Pacha es concebida como naturaleza aparente. Esta Pacha es secundaria ya que es "aprehendida en percepción". Esta segunda Pacha es, por lo tanto, el verdor y la suavidad que nos provoca percibir el entramado de elementos infinitesimales mencionados en el párrafo anterior.

Es importante recalcar que estas dos Pachas sólo existen de jure ya que, de facto, sólo existe una Pacha, pliegue cósmico, la cual es deliberadamente bifurcada para fines explicativos. Es que, como ya dije anteriormente, los casi quinientos años de contacto los han hecho entender para que el blanco no clasifique los puntos de vista de matriz africana como meras creencias equivocadas, se les tiene que explicar usando dualidades que conlleven al antropocentrismo. Por lo tanto, este primer paso de la explicación podría ser resumida de la siguiente manera:

\section{Pacha causal $\rightarrow$ Mente $\rightarrow$ Pacha aparente}

\section{MUNDO-PLIEGUE}

Después de haber aprehendido el movimiento de la bifurcación de la Pacha, pasé a un segundo punto en mi aprendizaje de la compleja concepción tocañera de la Pacha. En este segundo paso, me vi compelido a preguntarme por su origen. Sin embargo, ante mi pregunta ¿¿Cómo o cuándo se originó la Pacha?”, los Tocañeros respondían diciendo dónde la podía encontrar. Nunca escuché, de boca de los afrobolivianos, un mito de origen de la Pacha. El tío Juanchín, siempre que le preguntaba, argumentaba que eso no les preocupaba y que si quería escuchar cuentos sobre cómo se creó podía preguntarles a los aymarás o quechuas. "Lo importante es que ella existe y que gracias a ella nosotros podemos existir", me decía. Con el pasar del tiempo, me enseñaron y aprendí, que es casi imposible ubicar el origen de ella ya que ella es, en sí misma, principio y fin. Por ello, en lugar de que angustiarnos su origen, los Tocañeros nos proponen preocuparnos por saber dónde podemos encontrarla. 
Hasta ese momento, basándome en el movimiento de bifurcación de la Pacha que había aprehendido durante los primeros meses de mi estancia en Tocaña, yo sabía que había una Pacha que estaba afuera, naturaleza, inaprensible en su totalidad, y una Pacha que estaba dentro de mí, naturaleza aparente, la cual nacía de mi propia subjetividad. La Pacha se encuentra afuera y adentro, concluí precipitadamente. Sin embargo, gracias a la apertura y paciencia de los tocañeros, con el pasar de las vivencias pude entender que la Pacha no es del orden del dentro/fuera, sino de la figura/fondo.

Como argumentan los tocañeros, la Pacha se encuentra en el fondo, bien en el fondo, de cada uno de nosotros y de todo lo que conocemos. Cada ser, por más pequeño o grande que sea, tiene a la Pacha como su capa más profunda, como su asentamiento del cual deviene su individualidad. Por lo tanto, todo lo que existe es, en principio, un punto perfectamente definido de, y localizable en, la Pacha. Y si en principio todo es en la Pacha es porque la Pacha es y está en todo. Dicho de otra manera, cada uno de los seres con los que cohabitamos son un aquí-y-ahora, pedazos discretos e infinitesimales que se desprenden de esa eternidad espacio-temporal que es la Pacha. En suma, la Pacha habita en mí, así como yo habito en ella. Al entender esto pensé que había llegado "al fondo" del asunto por lo que llegué hasta a ensayar una primera definición de la Pacha. Pensé, hasta ese momento, que la Pacha podía ser definida como la infinita conjunción de múltiples aquí-y-ahora infinitesimales que en ella se han trazado, se siguen trazando y se trazarán.

Al compartir esta tentativa definición con los Tocañeros, estos no parecían tan convencidos ya que, desde la perspectiva que ellos ocupan, la división espacio-tiempo no es necesariamente marcada. Conversando con tío Juanchín y tío Mastuco aprendí que las cosas en la Pacha no pueden ser definidas como espacio-tiempo, ya que no son esencias, las cosas en la Pacha simplemente pasan, son procesos. Esto me llevó a preguntarme ¿Cómo hacer para pensar a la Pacha, la cual es un único ser indivisible, como la conjunción del espacio-tiempo? Es decir ¿cómo es posible concebir a la Pacha como un único ser, sin caer en la reificación de dos espacios discretos y separados?

Recapitulando mis conversaciones con la gente de Tocaña y otros antropólogos, pensé que una solución sensata a tan difícil emprendimiento era la de concebir la Pacha como un ser compuesto por una materialidad espacial traída a la vida a través del movimiento temporal. Tenía que dejar de ver a las cosas que me rodeaban como si 
estuvieran capturadas en fotografías, estáticas; y en su lugar, debía de verlas en movimiento, como una película. Era como si, en principio, tuviéramos una imagen estática de la Pacha causal, aquel material externo que causa nuestra percepción, a la cual se le insiere el tiempo. Esta operación la dotaría de vida, tornándola en aquello que Gilles Deleuze denomina imagen-movimiento. Todo este proceso provoca que la Pacha causal devenga en la Pacha aparente, esa que aprehendemos en percepción.

$\mathrm{Al}$ exponer estas ideas iniciales en eventos antropológicos, algunos colegas me advirtieron de que en mi entendimiento de la Pacha había cierto peligro de deificar una concepción monista y totalizadora. Para contrarrestar este riego, me sugirieron, podía enfatizar el hecho que las dos Pachas no designaban dos provincias ontológicas diversas (Pacha causal y Pacha aparente) más sí un contexto relacional que unía a ambas. "La Pacha es la que nos da el soplo de vida que nos hace ser cada quién", me explicó awicho Chan Chan. Esta sugerencia, la cual consideré interesante y osada, me llevó a prestar atención a algunos puntos que antes no había notado. La Pacha no sólo era lo material, o las abstracciones subjetivas que se adhieren a lo material. La Pacha también se encontraba en aquello que hacía que existiera la diferencia.

La Pacha radicaba, según los tocañeros, también en aquello que llamaban cultura, en sus modos de vida. Esto me llevó a pensar que la Pacha, en lugar de ser aquella materialidad inicial que posteriormente permite la creación de subjetividades, sería mejor definida como la conjunción no aditiva de múltiples puntos de vista, los cuales al ser ocupados por individuos, los tornan sujetos. Es decir, la pacha no es la suma del punto de vista tuyo, más el punto de vista mío, más el punto de vista de fulano, de sutano y de mengano. Diferentemente, la Pacha es la infinitud de puntos de vista a ser ocupados.

Entablando diálogos con el perspectivismo amerindio, conseguí ver el polo relacional de la concepción Tocañera de la Pachamama que, hasta ese momento, me había sido esquivo. Retomando mis notas iniciales, partí del hecho de que la Pacha está en todos nosotros, y nosotros estamos en la Pacha. Es decir, partí del entendimiento de que la Pacha es la capa más profunda de todos los seres que en ella habitan. Esto me condujo a pensar, por consiguiente, que cada uno de los individuos creados por la Pacha no era un mero punto discreto de una malla espacio-temporal sino que era, en sí, un espejo vivificado de la Pacha entera, vista desde un punto de vista determinado. Esto es, la Pacha no sólo es fondo sino también, siempre simultáneamente, figura. Es como cuando nos miramos en 
espejo bastante pequeño. Dependiendo de la posición del espejo, podremos ver tan sólo una pequeña parte de nuestro cuerpo. Supongamos que debido a la ubicación del espejo podamos ver tan sólo el dedo gordo de la mano derecha. En el acto de ver al espejo, nosotros (como un todo) nos tornamos figura mientras que el espejo se torna fondo. Sin embargo, una vez que percibimos aquella imagen reflejada por/en el espejo, nosotros (como un todo) pasamos a ser fondo, mientras que el dedo gordo (el cual es nuestro, pero al mismo tiempo deviene individual) pasa a ser figura, en la cual está encapsulada la misma esencia que nos hace (un todo).

Sé que el ejemplo anterior es bastante mundano, pero creo que nos sirve de ayuda ya que algo similar (salvando las distancia) pasa con la Pacha. Esta antecede a los sujetos que ella crea, a pesar de que ella no pueda existir fuera de estos sujetos que la expresan. Por ende, la Pacha es un pliegue cósmico formado por las partículas del polvo del cual todos los seres venimos y en el cual todos deveniremos para, una vez más y así hacia la eternidad, volver a recrearnos mientras la recreamos. "De la tierra venimos y a la tierra volveremos", dicen frecuentemente los Tocañeros. Por ello, pensé, la Pacha puede ser vista como un mundo-pliegue del cual nace la diferencia en sí. Un mundo-pliegue en el que todo, en lugar de ser, acontece... O mejor dicho, la Pacha más que un ser es un aconteser.

Leyendo diversos artículos académicos y conversando con colegas antropólogos, fue que me percaté que las maneras de acomodar y explicar las relaciones entre las Pachas causal y aparente, fluctuaban, entre "los dualistas que aceptan a la materia y mente en igualdad de condiciones, y las dos variedades de monistas, aquellos que colocan la mente dentro de la materia y aquellos que colocan la materia dentro de la mente" (Whitehead, 1929). Hasta ese momento parecía que para definir la Pacha no podría escapar de las cuatro ontologías propuestas por Philippe Descola (1997); o me quedaba en las explicaciones dualistas, las cuales remitían a las ontologías totemistas y analista, o tomaba las vertientes monistas, las cuales refinaban al naturalismo (que coloca la mente dentro de la materia) y el animismo (que coloca la materia dentro de la mente). Pero ninguna de estas opciones me satisfacía ya que fuera como fuese y, a pesar de las divergencias conceptuales, el punto en común que teníamos todos aquellos antropólogos que buscábamos aprehender la noción tocañera de Pacha era la de unir, siempre a posteriori, 
dos facetas bifurcadas de una única Pacha a priorística, o parafraseando a Didier Debaise, conectar una conjetura (Pacha causal) a un sueño (Pacha aparente)” (2017, p. 26).

\section{UNO MÚLTIPLE}

Con los tocañeros, así como con Isabel Stengers, había aprendido que era sumamente importante pensar desde el medio, siempre, entre África y los Andes, desde tocaña, entre la esclavitud y la libertad, desde la experiencia de las poblaciones menores, entre lo negro y lo indígena, desde el punto de vista de los afroyungueños. Hablar desde el medio, entendido como un entre dos y como un oikos... hablar desde medio siempre siendo un uno múltiple.

Pensar desde el medio, me llevó a adentrarme aun más en la definición de la Pacha. Fue así como surgió la siguiente pregunta: ¿Cómo hacer para entender a la Pacha como un uno múltiple? Al preguntarle esto a tío Juanchín, me hizo comprender que la Pacha es como cualquier otro Tocañero. La única diferencia es que la Pacha era un tocañera tan grande, que en ella también se encapsulaba todo lo que sabemos que conforma el mundo que habitamos, desde el Perolani hasta un grano de arena. Es un ser tan grande que nunca la conoceremos enteramente, como fondo. Por ello, siempre que nos topamos con ella, nos topamos con sólo parte de ella, como figura. Así, y pactando con la ontología propuesta por Whitehead, es que me animo a afirmar la definición mínima de la Pacha, desde el punto de vista de los tocañeros, "es todo aquello que observamos en percepción mediante los sentidos" (Whitehead 1920, p. 28). Todo aquello de lo que somos conscientes que existe incluso cuando no tengamos las palabras precisas para denominarlo.

Y si la Pacha es incluso aquello que no puede ser definido por palabras, entonces, en lugar de pensar con la cabeza, era necesario sentirla con todo el cuerpo, creando vínculos estrechos entre mi cuerpo con el ambiente, entre mi ethos y el oikos. Para ellos, debía de salir del registro de la esencia y el ser para, una vez mas, adentrarme en el orden del devenir y el aconte-ser. Así se me hizo claro que lo que conocemos de la Pacha lo conocemos a través de nuestra experiencia subjetiva. Por ello, comprendí, era fundamental colocar la siguiente restricción propuesta también por Didier Debaise: se tiene que tomar a la experiencia en serio, sin adicionar ni substraer nada de ella. "A la 
Pacha tenemos que observarla con todo el cuerpo, es la única manera de verla", me decía tía Raymunda en Tocaña. La Pacha no se ve con los ojos, la Pacha se siente con los ojos y con todo el cuerpo, al mismo tiempo.

Pero sentir en este caso debe de ser tomado, simultáneamente, como verbo y sustantivo, tanto en la forma en que expresa certeza subjetiva como en la forma en la que expresa tonalidad afectiva. "Siento peligro porque siento que El Tío debe andar cerca", me explicó una vez tío Juanchín. Esto nos puede servir como ejemplo para entender, en este caso, que el sentir está estrechamente ligado al aprehender. No sólo siento lo tangible, como una piedra que golpea mi cuerpo, sino también lo desconocido, lo posible que aún no es. Y siento los dos de la misma forma. Sentir es, por lo tanto, ser capaz de formar parte de un proceso de afectación en el cual se capturan cosas de sujetos otros, cosas de un pasado no pasado (Stolze Lima, 2018) las cuales pueden ser actualizadas. En palabras de Debaise, "es como si cada ser tuviera una doble existencia: Una que departe de su propia perspectiva, su actividad presente, y esa otra que departe de su ser capturado en actos de sentir posteriores" (2017, p. 46).

Para ver a la Pacha, entonces, más que buscar respuestas que giren en torno a la idea de ser, debemos de centrarnos en la idea Tardiana de tener. Tengo dolor, tengo miedo, tengo un perro, tengo hijos. Sujeto es aquel que tiene o que siente (lo aprehendido) y no aquel que es (la substancia). La idea de ser nos estabiliza y substancializa, alejándonos de la Pacha. El tener, en cambio, nos trae a ella, nos conecta a ella, nos obliga a establecer una relación en y con ella, ya que en cada acto de aprehensión, la capturamos, la sentimos, a partir del punto de vista específico que ocupamos.

También aprendí que aunque la Pacha sea de todos los tiempos, desde el punto de vista de los tocañeros, solo es posible aprehenderla, sentirla, en el tiempo presente; esto es, como un pasado no pasado. Al capturarla en el presente, actualizamos, a nuestra manera, a nuestro sentir, toda su existencia pasada, la cual nos puede ayudar a revitalizar mundos y territorios posibles que aun no se han tornado parte de nuestra realidad actual. Y ya que cada ser interpreta, quiero decir, aprehende, a la Pacha de forma singular, nunca se tendrá una definición exacta y consensuada de lo que es la Pacha.

Inclusive, a pesar de tener la seguridad total de que la Pacha seguirá existiendo en su infinitud, no podemos saber a ciencia cierta de qué manera lo hará. Cada uno de los sujetos que en ella habitan se encargarán de transformarla mientras ella también los va

Revista da ABPN • v. 12, n. Ed. Especial - Caderno Temático: "Geografias Negras" • abril de 2020, p. 418-434 
transformando. Por eso dicen, "de la Pacha venimos y a la Pacha vamos". Y así, al llegar al final de nuestras transformaciones nuestro cuerpo regresará al Aka Pacha (mundo de aquí) para que el tiempo de las transformaciones prosiga; nuestro alma (punto de vista) se difuminará yendo al Axal Pacha (mundo de arriba) en el cual habitará hasta la eternidad; y, finalmente, nuestro ánimo (fuerza vital), emprenderá el viaje al Manqha Pacha (mundo de abajo), para que desde el pasado vuelva surgir de forma diferente. En suma, aquello que fue de este mundo, retornará a todos los otros mundos, para después volver a tornarse real en otros aconte-seres, que al igual que nosotros habitarán la Pacha.

\section{ACONTE-SERES (O A MODO DE CONCLUSIÓN)}

A pesar de que cada uno de nosotros, individuos, sea producto de actualizaciones del pasado somos presente, y, a pesar de estar en procesos de devenir constante, aun seguimos siendo los mismos, hasta que nos llegue el tiempo del fin de nuestras transformaciones. Somos múltiples y a la vez únicos, todos somos diferencia, y cada uno es diferencia diferenciada. Por ejemplo, en el Perolani vi que los árboles que buscábamos eran todos iguales... delgados, altos, rectos, blancos. Pero de cerca cada uno tenía características diferentes. Unos son más suaves, unos más blancos, unos más húmedos, unos más cálidos. Todas estas cualidades hacen al árbol, de la misma forma que lo hace su materialidad. La diferencia es que estas cualidades no sólo se encuentran en el árbol, sino que hacen parte de esa serie de posibles que se han injertado en los árboles y en otros seres, dándoles características específicas a cada uno.

Estas cualidades son elementos adverbiales y no sustantivos, es decir, no son meras características de nuestro ser sino que son elementos capaces de afectar nuestro devenir. Además, así como la Pacha, estas cualidades, por pequeñas que sean, son abstractas pero reales, eternas pero creativas, creativas más no creadores, son, en palabras de Whitehead, “objetos eternos”. Somos únicos porque, a pesar de ser reflejos del pasado, nuestro ambiente en contrapunto con nuestro ethos, le da la posibilidad a estos "objetos eternos" de injertarse en los procesos de devenir que nos hacen y rehacen constantemente. Por ello, dicen en Tocaña, que en el fondo todos compartimos muchas cosas con otras personas e incluso con los dioses, pero a pesar de ello, cada uno tiene su diferencia propia. 
Este contrapunto entre ambiente y sujeto me llevó a ver que, lo que desde un punto de vista es sujeto, de otro punto de vista se torna parte del ambiente. O, para tejerlo con lo erguido a lo largo del texto, lo que desde un punto de vista es figura, desde otro punto de vista puede ser fondo, y viceversa. Es en este juego de claroscuros que podemos encontrar a la Pacha. Por ello decimos que uno es la Pacha mientras que la Pacha también es uno. Todo pasa en encuentros y en capturas, todo pasa a través de la relación del ethos con el oikos, y viceversa. La Pacha propone y la relación entre sujetos dispone. Por eso, el que yo, usando mi machete, sea capaz de cortar aquel árbol, no significa que yo con el mismo machete después de diez años sea capaz de cortarlo, o que yo también pueda cortar otro árbol de la misma especie en otro ambiente y con otro machete. Todo encuentro, gracias a esta insistencia de posibles otros, resulta siempre en eventos diferentes. Esto no solo se da en el Aka Pacha, sino también en el Axal Pacha y el Manqha Pacha, no sólo se da en este tiempo, sino en todos los otros tiempos, no sólo se da en este espacio, sino en todos los otros espacios, no sólo se da en este nivel, sino también en lo infinitesimal y en lo transfinito. Es decir, esta insistencia de posibles se da a todo nivel de la eterna Pacha, en la que nada se puede predecir o explicar a priori, sino más bien todo acaece $a$ posteriori. "En el Perolani es peligroso porque la Pacha puede estar en el árbol que uno corta, o en una hoja del árbol, o puede estar vigilándote desde cerquita”, explicaba tío Mastuco.

Es por ello que es difícil responder a la pregunta ontológica ¿qué es la Pacha? Vuelvo a repetir, a la Pacha más que pensarla tenemos que experimentarla, capturarla, sentirla. Y si ese es el requisito principal, entonces debemos, una vez más, desacelerar y, más que intentar dar una definición general de la Pacha, debemos limitarnos a definirla a partir de nuestro punto de vista. Por ello, desde la perspectiva de los Tocañeros, la Pacha es, complementando lo anteriormente argüido, un conjunto de eventos pasajeros que observamos, en percepción, a través de los sentidos. Eventos en los cuales se intrincan simultáneamente tiempo, espacio, y sujetos múltiples, todo en un ensamblaje de relaciones establecidas tanto en lo infinitesimal como en lo transfinito. Es así que junto a los Tocañeros aprendí que la Pacha, así como todo lo que en ella habita, nunca simplemente está, sino que siempre está siendo. Por ello, no se trata de seres sino de aconte-seres. 
En otras palabras, los sujetos y eventos comparten la misma categoría ontológica. Más que seres estables, somos eventos, acontecimientos compuestos por ensamblajes de otros eventos menos y mayores. Nuestro cuerpo es un evento, nuestra alma es un evento, nuestro espíritu es evento. Todo el tiempo estamos cambiando, pero a pesar del cambio todo seguimos siendo. Al igual que la Pacha somos aconte-seres en constante devenir. Somos igual que la Pacha, porque somos un espejo de ella, sólo que a diferente escala. Así comprendí que la Pacha radica en todo. Desde lo más pequeño que no llega a ser la nada, lo infinitesimal, hasta en lo más grande que no llega a ser el todo, lo transfinito. En todos esos lugares está la Pacha. Por ello es que se afirma que la Pacha es como un pliegue que, a pesar de ser inconmensurable e omnipresente, puede también habitar en cada uno de nosotros. Al concebir a la Pacha como pliegue entre lo infinitesimal y lo transfinito, así como un pliegue entre lo espacial y lo temporal, comprendí que, a diferencia de las metafísicas que parten de la bifurcación de la naturaleza, para los tocañeros en la Pacha no existen las discontinuidades ni naturales ni culturales.

Por ello tampoco existe una división ontológica entre lo abstracto y lo material, entre el espíritu y el cuerpo. En la Pacha, espíritu y cuerpo comparten la misma condición y son inherentes el uno al otro, son las dos caras de un mismo pliegue. Ambos se encuentran en posiciones contiguas, ambos son constituidos por el polvo cósmico de mónadas concatenadas que catalizan nuestros procesos integradores y diferenciadores de devenir. Por ello, se puede afirmar que nuestro cuerpo y espíritu no son dos elementos diferenciados que nos constituyen. Ellos, al igual que el mundo que habitamos, son pliegues y repliegues de sí. Esto genera que, aunque sea menos obvio, cada uno de nosotros podamos ser, al igual que el Perolani, si las condiciones del ambiente lo permiten, ser una puerta cósmica a la Pacha en su plenitud.

La Pacha, así, puede ser entendida como un aconte-ser de confluencia y transfluencia (Bispo dos Santos 2015, p. 89). Es decir, un aconte-ser que, aproxima extensivamente sujetos sin homogeneizar intensivamente sus fuerzas y materiales, al mismo tiempo que separa intensivamente fuerzas y materiales sin reificar una diversidad extensiva de sujetos. La Pacha, por lo tanto, es un ensamblaje de fuerzas y materiales que, como dice Antonio Bispo dos Santos, "no todo lo que junta lo mezcla y no todo lo que mezcla lo junta" (Bispo dos Santos 2015, p. 89). Más que un agujero negro, es un agujero de gusano (wormhole). Esto es, un pliegue cósmico que, en lugar de ser un posible

Revista da ABPN • v. 12, n. Ed. Especial - Caderno Temático: "Geografias Negras”• abril de 2020, p. 418-434 
resultado de una línea de fuga fallida, se presenta como una condensación infinita de fuerzas espacio-temporales la cual sirve como portal conector de cosmos múltiples. Pliegue cósmico en el cual no existe discontinuidad alguna entre un dentro y un afuera, entre el tiempo y espacio, entre lo infinitesimalmente pequeño y la inmensidad transfinita. La Pacha, por ende, es una especie de vórtice cósmico en el que se todo se encuentra conectado a través de la condensación de fuerzas centrípetas y centrifugas que propulsan procesos de devenir constantes e infinitos.

\section{REFERÊNCIAS BIBLIOGRÁFICAS}

BISPO, A. Colonização, quilombos, modos e significados. Brasilia: INCT, 2015.

DEBAISE, D. Nature as event: The lure of the possible. Trans. Michael Halewood. Durham, N.C.: Duke University Press, 2017.

DESCOLA, P. The spears of twilight: life and death in the Amazon jungle. London: Flamingo, 1997.

WHITEHEAD, A. Process and Reality: An Essay in Cosmology. David Ray Griffin and Donald Sherburne eds. Gifford Lectures of 1927 - 28. New York: Free Press, 1929.

WHITEHEAD, A. The concept of nature. The Tarner Lectures Delivered in Trinity College: Cambridge, 1919.

Recebido 20/02/2020

Aceito em 30/03/2020 